\title{
EXPERIMENTAL INVESTIGATION OF GAS HYDRATE PRODUCTION AT INJECTION OF LIQUID NITROGEN INTO WATER WITH BUBBLES OF FREON 134A
}

\author{
Anton $V$. Meleshkin ${ }^{1}$, Ivan $V$. Mezentsev ${ }^{1, *}$, and Dmitriy $S$. Elistratov ${ }^{1}$ \\ ${ }^{1}$ Kutateladze Institute of Thermophysics SB RAS, Novosibirsk, Russia
}

\begin{abstract}
The hydrodynamic processes during the injection of the cryogenic liquid into the volume of water with bubbles of gas freon 134a are studding experimentally. A processes during the explosive boiling of liquid nitrogen in the volume of water are registered. Video recording of identified gas hydrate flakes formed during this process is carried out by high speed camera. These results may be useful for the study of the new method of producing gas hydrates, based on the shock-wave method.
\end{abstract}

\section{Introduction}

There are many methods for intensifying the process of hydrate formation: intensive mixing of water saturated with dissolved gas; finely dispersed atomization of a water jet; vibration and ultrasonic impact on the two-phase systems, etc. The main disadvantage of the proposed methods is the low rate of gas hydrate formation and, therefore, low performance of the systems based on these methods. The Institute of Thermophysics SB RAS is involved into artificial gas hydrate production by the shock-wave method since 2000 . In 2003, the method for producing gas hydrates has been proposed (RF patent No. 2270053). The essence of this invention is as follows. The compressed and cooled gas is supplied into the reaction vessel filled with water, which is under the static pressure and the temperature below the equilibrium temperature of hydrate formation. The gas pressure is slightly higher than the pressure in the reaction vessel, and the gas temperature equals the temperature of water in the vessel. Gas is mixed with water. The pressure shock waves with the amplitude of up to the hundreds of atmospheres are generated in the gas-liquid medium. The shock waves can be generated by the electromagnetic pulse emitters, pneumostrikers and other devices $[1,2]$. When the shock wave propagates in the gas-liquid medium, the pressure increases and the gas phase crushes throughout the reaction vessel due to weak dissipation of the wave. Therefore, the degree of medium metastability and the number of nucleation sites of gas hydrate increase, the size of gas inclusions decreases, interfacial surface increases, the relative velocity of gas inclusions in liquid increases, and liquid motion

* Corresponding author: mezn@itp.nsc.ru 
becomes more turbulent. All these phenomena accelerate the mass transfer process at the interface and, hence, intensify the process of hydrate formation.

This paper presents the results of studying the shock-wave method of gas hydrate production by injecting liquid nitrogen. The authors of [3] studied the process of nitrogen supply into a vessel of a small volume $(140 \mathrm{ml})$. The volume of injected liquid was about 2 $\mathrm{ml}$. For example, at introduction of liquid nitrogen into water ( 7 bars), the amplitude of the pressure jump reaches 14 bars in 5 seconds. Previously, we have carried out the experiments on entering a jet of liquid nitrogen in the water volume [4]. The maximal amplitude of the pressure jump in these experiments was 53 bars, and the rate of pressure rise was $567 \mathrm{bar} / \mathrm{s}$. These results were a part of the works required for the development of a new method of gas hydrate production, based on the shock-wave method.

\section{Experimental setup and methods}

Gas hydrate production was studied at the experimental setup with the viewing windows along the entire height of the working section. The working section is a parallelepiped with the height of $740 \mathrm{~mm}$ (cross-section of $150 \times 150 \mathrm{~mm}$ ) made of stainless steel with the wall thickness of $15 \mathrm{~mm}$. Two viewing windows in the working section, allowed us to fix the processes occurring at applying a jet of liquid nitrogen into water, and namely to carry out the high-speed optical measurement. Gas was supplied through the bubble generator from the bottom of the setup.

Liquid nitrogen was fed into the working section as a jet through the injector (fig. 1). The volume of injected liquid nitrogen was $28 \mathrm{ml}$.

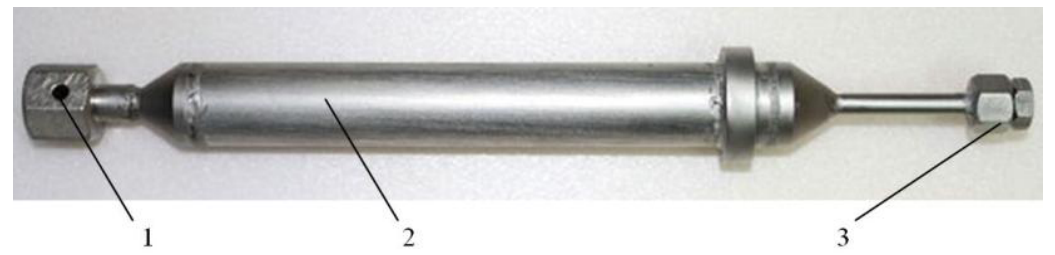

Fig. 1. General view of the injector: 1 - ring slot, 2 - injector chamber, 3 - input unit.

The storyboard of resulting high-speed shooting of liquid nitrogen injection into water saturated with bubbles of Freon gas is shown in fig. 2. In the first frame, the working section saturated with the bubbles of Freon R134a can be clearly seen; in the second frame, liquid nitrogen is injected. In the third and fourth frames, we can see rapid mixing of the working section due to liquid nitrogen boiling in water. In the fifth and sixth frames, the working section after liquid nitrogen boiling is presented. The gel-like gas hydrate flakes, whose generation rate is high, can be clearly seen in the frames; this gives an order advantage of the shock-wave method over all known analogical methods.

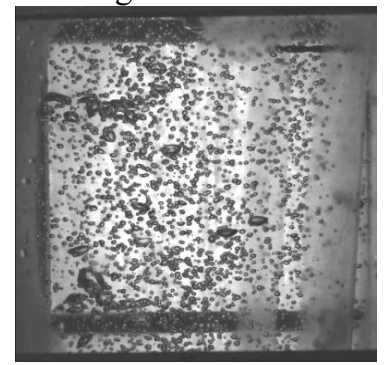

$0 \mathrm{~ms}$

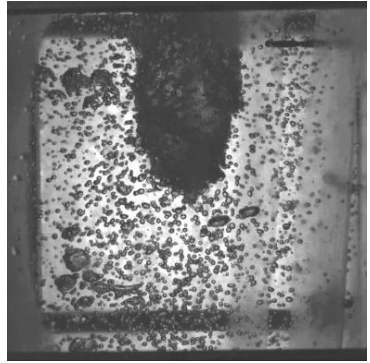

$38 \mathrm{~ms}$

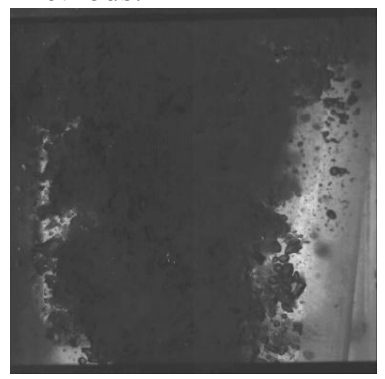

$594 \mathrm{~ms}$ 


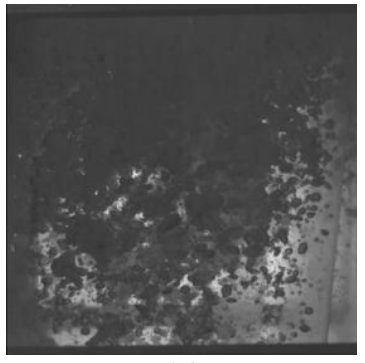

$734 \mathrm{~ms}$

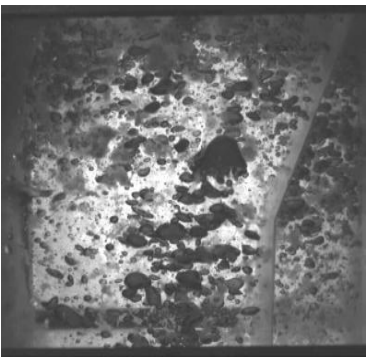

$1146 \mathrm{~ms}$

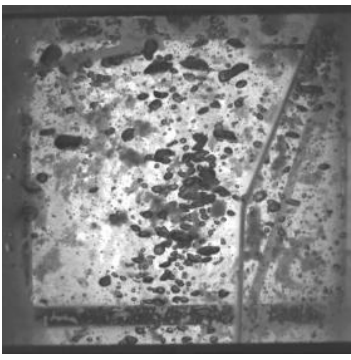

$2580 \mathrm{~ms}$

Fig. 2. Frames of high-speed recording of the process of liquid nitrogen injection (10 $000 \mathrm{fr} / \mathrm{s})$.

This method of gas hydrate production does not require the additional external sources of the pulse pressure. Here, pulsating (oscillating) gas and steam bubbles are the powerful tool for pressure pulse generation; they can create powerful pressure pulses, which destroy even steel. To initiate bubble pulsation, it is necessary to give a significant initial rate to the interface, for example, by micro bursting. For this purposes, a jet of liquid nitrogen, which can be overheated to the spinodal temperatures, as a pure cryogenic liquid. Subsequent heating causes explosive boiling of a part of liquid nitrogen and disperses the boundary of a gas cavity.

During the experimental studies, the pressure amplitude at liquid nitrogen boiling in water with Freon bubbles, whose maximal value was 4.2 bars, was measured. This pressure jump allowed us to reach the metastable equilibrium zone of Freon R134a gas hydrate.

\section{Conclusion}

This method of gas hydrate production does not require the additional external sources of the pulse pressure. Here, pulsating (oscillating) gas and steam bubbles are the powerful tool for pressure pulse generation; they can create powerful pressure pulses, which destroy even steel. To initiate bubble pulsation, it is necessary to give a significant initial rate to the interface, for example, by micro bursting. For this purposes, a jet of liquid nitrogen, which can be overheated to the spinodal temperatures, as a pure cryogenic liquid. Subsequent heating causes explosive boiling of a part of liquid nitrogen and disperses the boundary of a gas cavity.

During the experimental studies, the pressure amplitude at liquid nitrogen boiling in water with Freon bubbles, whose maximal value was 4.2 bars, was measured. This pressure jump allowed us to reach the metastable equilibrium zone of Freon R134a gas hydrate.

\section{Acknowledgments}

The work was financially supported by the Russian Science Foundation 15-19-10025.

\section{References}

1. V.E. Dontsov, A.A. Chernov Doklady Physics 54, 4 (2009)

2. V.E. Nakoryakov, V.E. Dontsov, A.A. Chernov, Doklady Physics. 51, 11 (2006)

3. H. Clarke, A. Martinez-Herasme, R. Crookes, D.S. Wen, Int. J. Multiphase Flow 36, 4 (2010)

4. V.E. Nakoryakov, A.N. Tsoi, I.V. Mezentsev, A.V. Meleshkin J. Eng. Thermophys. 23, 1 (2014) 